\title{
STABILITY IN A SYSTEM OF DIFFERENCE EQUATIONS*
}

BY

DEAN S. CLARK

University of Rhode Island

0. Introduction. It is well known that solutions to difference equations can behave differently from those of their differential-equation analog [1], [6], but the following presents a particularly weird instance of this fact.

Example 1. Let $x_{n+1}=x_{n}-M\left(\operatorname{sgn} x_{n}\right) \sqrt{\left|x_{n}\right|}$, where $M$ is a large positive constant and $\operatorname{sgn} x=1$ if $x \geqslant 0, \operatorname{sgn} x=-1$ otherwise. Solutions to this equation in continuous time, $\dot{x}(t)=-M(\operatorname{sgn} x(t)) \sqrt{|x(t)|}$, show convergence to the origin from all initial positions. In contrast, it doesn't matter how close the initial value $x_{0} \neq 0$ is to zero, succeeding iterates $x_{n}$ oscillate away to great distances from the origin. For these initial values $\lim _{n \rightarrow \infty}\left|x_{n}\right|=$ $M^{2} / 4$. At the same time, there are initial positions arbitrarily far from zero such that $x_{n}$ lands on the origin in a finite number of steps! For example, if $x_{0}=\left(\frac{3+\sqrt{5}}{2}\right) M^{2}$, then $x_{1}=M^{2}, x_{2}=x_{3}=\cdots=0$.

It takes only a small adjustment to bring the asymptotic behavior for both discrete and continuous-time equations into harmony. Replace the large $M$ with small $\varepsilon>0$. Hence,

Definition 1. Let the solution $X_{n}(\varepsilon)$ to a difference equation be parametrized by $\varepsilon>0$. We say the solution exhibits near-convergence to zero if

$$
\lim _{\varepsilon \rightarrow 0} \varlimsup_{n \rightarrow \infty}\left|X_{n}(\varepsilon)\right|=0 .
$$

This paper deals with the problem of near-convergence for systems of difference equations which are under perturbation. Our systems will be general enough to model a number of interesting situations.

Example 2. Take the simplest linear difference equation $x_{n+1}=(1-\varepsilon) x_{n}, 0<\varepsilon<1$, and perturb it: $\bar{x}_{n+1}=(1-\varepsilon) \bar{x}_{n}+\varepsilon \sin n$. Such a perturbation is critical in the sense that it can be made arbitrarily small by taking $\varepsilon$ small, but this has the effect of diminishing the attractive force of the origin. When $\varepsilon=0$ there is no perturbation and no convergence

\footnotetext{
* Received March 9, 1986.
} 
to zero either. We ask if solutions $\bar{x}_{n}$ show near-convergence in the sense of (1), and the answer is that they do, from all initial positions. This is easy to show by elementary methods. A seemingly innocent modification destroys the property. Let $y_{n+1}=(1-\varepsilon) y_{n}$ $+\varepsilon \sin \sqrt{n}$. The trajectories $y_{n}$ can be made to travel arbitrarily far from the origin, no matter how small $\varepsilon$ becomes, by multiplying $\sin \sqrt{n}$ by a large constant $M>0$. We prove this fact in the Appendix using the basic interpolation method introduced in (10) below.

Example 3. Perturb the simplest equation randomly: $u_{n+1}=(1-\varepsilon) u_{n}+\varepsilon \xi_{n}$, where $\xi_{n}=1$ if a fair coin-toss shows "heads" on the $n$th toss, $\xi_{n}=-1$ if it shows "tails". We ask about near-convergence of solutions $u_{n}$ and the answer again is that it is destroyed. Because we are unable to describe the paths of $\xi_{n}$ analytically a theory must be developed to explain this breakdown.

Remark. The three perturbations in Examples 2 and 3 all have something in common, namely a negligible long-run average value:

$$
\frac{1}{n} \sum_{j=1}^{n} \sin j, \quad \frac{1}{n} \sum_{j=1}^{n} \sin \sqrt{j}, \quad \frac{1}{n} \sum_{j=1}^{n} \xi_{j} \rightarrow 0
$$

as $n \rightarrow \infty$, the last limit with probability one (the strong law of large numbers). Yet, only the first perturbation, $\sin n$, preserves near-convergence. Why?

1. Difference equations under perturbation. One reason why difference equations model certain situations more accurately than differential equations is that decision-making, which takes discrete time, may be needed to determine the adjustment $X_{n+1}-X_{n}=\Delta X_{n}$. Moreover, there are occasions when not only the direction, but also the speed or intensity of adjustment is the subject of policy. Armed nuclear opponents, for example, think not only about building up force or cutting back, but also about restraining or intensifying their intended reactions. They also think about the unpredictable behavior of their rivals, hence the unspecified perturbations. For use of the term "speed of adjustment" (in the sense of coefficient $\varepsilon$ in Examples 1-3) in the context of a dynamic Cournot duopoly model, see [4, p. 227]. Additional remarks on the modelling and simulation aspects appear in the Appendix.

In this paper we study the system of difference equations, with perturbation term $\Psi_{n}\left(X_{n}\right)$,

$$
X_{n+1}=X_{n}+E_{n}\left(G_{n}\left(X_{n}\right)+\Psi_{n}\left(X_{n}\right)\right), \quad n=0,1, \ldots,
$$

where $G_{n}: \mathbf{R}^{k} \rightarrow \mathbf{R}^{k}, G_{n}(0)=0$, and $E_{n}=\operatorname{Diag}\left(\varepsilon_{n}^{1}, \varepsilon_{n}^{2}, \ldots, \varepsilon_{n}^{k}\right)$ is the diagonal matrix of adjustment speeds $\varepsilon_{n}^{j}>0, j=1, \ldots, k$.

Remark. Equation (2) is just as general as the nonlinear equation $X_{n+1}=X_{n}+$ $E_{n} F_{n}\left(X_{n}, \Phi_{n}\right)$ with perturbation $\Phi_{n}$. To transform this into $(2)$, let $G_{n}(\cdot)=F_{n}(\cdot, 0)$, $\Psi_{n}=F_{n}\left(X_{n}, \Phi_{n}\right)-F_{n}\left(X_{n}, 0\right)$.

Notational convention. $O(x)$ always signals a numerical value satisfying $|O(x)| \leqslant M|x|$ for a sufficiently large constant $M$ independent of $x$.

Before adapting the classical definition of stability to (2), some remarks on the coefficients $\varepsilon_{n}^{j}$. Now they are free to vary over time. However, we know from the Introduction that interesting stability results will be impossible unless they are kept small. 
This is accomplished by assuming, henceforth, that $\left|\varepsilon_{n}^{j}-\varepsilon\right|=O\left(\varepsilon^{2}\right)$ for all $j$ and $n$. This amount of variability may seem marginal, still, we feel it's worth knowing how far $\varepsilon_{n}^{j}$ can be from known, small $\varepsilon>0$.

DeFinition 2. We say that the zero solution (of (2), without the perturbation) is uniformly stable under perturbation if, given any $\eta>0$, there exist $\delta(\eta)>0$ and $\varepsilon^{*}(\eta)>0$ sufficiently small so that $\left|X_{n_{0}}\right|<\delta$ and $0<\varepsilon<\varepsilon^{*}$ imply $\left|X_{n}(\varepsilon)\right|<\eta$ for all $n \geqslant n_{0} \cdot|\cdot|$ denotes the Euclidean norm.

Our goal is to develop, and interpret, necessary and sufficient conditions on the perturbations for stability in system (2) in the sense of Definition 2. Of course, problems of stability in difference equations and problems of how an otherwise stable system reacts to perturbation are not new. LaSalle [7] has an extensive bibliography. Liapunov methods, which we use, have been used before [3], [5]. However, the type of perturbation we consider is new. In the past, stability criteria, say for the differential equation $\dot{x}=f(t, x)$ $+h(t, x)$, have required that $h(t, x)$ be small in $x$. But our perturbations $\Psi_{n}\left(X_{n}\right)$ depend on the solutions $X_{n}$ and are potentially big. The only way to dampen their impact is to take $\varepsilon$ small, simultaneously reducing the "traction" of the origin. The possible destabilizing effect was mentioned in Example 2.

Lemma 1, below, introduces criterion (3), which will become the necessary and sufficient condition we seek. The purpose of the lemma is to help interpret the meaning of (3) in terms of the ergodic and boundedness properties of $\left\{\xi_{n}\right\}_{n=0}^{\infty}$.

Lemma 1. Let $\left\{\xi_{n}\right\}_{n=0}^{\infty}$ be a sequence of elements in a normed linear space. Suppose that for any $\eta>0$ there is a sufficiently large $c(\eta)>0$ so that, for all $m, n \geqslant 0$,

$$
\left\|\sum_{j=m}^{n-1} \xi_{j}\right\|-\eta|n-m| \leqslant c(\eta) .
$$

Then, the averages satisfy

$$
\lim _{N \rightarrow \infty}\left\{\sup _{n \geqslant 0} \frac{1}{N+1}\left\|\sum_{j=n}^{n+N} \xi_{j}\right\|\right\}=0,
$$

and

$$
\sup _{n \geqslant 0}\left\|\xi_{n}\right\|<\infty
$$

Moreover, (4)-(5) implies (3).

Proof. ((3) $\Rightarrow(4)-(5))$. Divide both sides of (3) by $|n-m|$ taken large enough so that $c(\eta) /|n-m|<\eta$. Tris implies (4). Then, set $n=m+1$ to get (5).

((4)-(5) $\Rightarrow(3)$ ). If, given $\eta>0$, $\sup _{n \geqslant 0}\left\|\xi_{n}\right\|<B(\eta)<\infty$ and (4) holds, then, with sufficiently large $N(\eta)$ we have

$$
\left\|\sum_{j=n}^{n+r} \xi_{j}\right\| \leqslant \eta(r+1)+B(N+1), \quad n, r \geqslant 0,
$$

which is equivalent to (3). 
There are many examples of such sequences $\left\{\xi_{n}\right\}_{n=0}^{\infty}$, but first we show that (3) follows easily as a necessary condition for stability in (2).

Theorem 1. Suppose that $\left\{G_{n}(\cdot)\right\}_{n=0}^{\infty}$ is equicontinuous at the origin and that the zero solution of (2) is stable in the sense of Definition 2. Then, for any $\eta>0$, there exist $\delta(\eta)>0$ and $\varepsilon^{*}(\eta)>0$ such that $\left|X_{n_{0}}\right|<\delta$ and $0<\varepsilon<\varepsilon^{*}$ imply

$$
\left|\sum_{j=m}^{n-1} \Psi_{j}\right|-\eta|n-m| \leqslant \eta / \varepsilon, \quad 0 \leqslant n_{0} \leqslant m, n \text {. }
$$

Proof. First we estimate the size of $\varepsilon\left|\Psi_{n}\right|$. Let $\Delta X_{n}=X_{n+1}-X_{n}$. With respect to a typical vector component (lower-case letters), (2) implies

$$
\varepsilon_{n} \psi_{n}=\Delta x_{n}-\varepsilon_{n} g_{n}\left(X_{n}\right) \text {, so } \varepsilon_{n}\left|\psi_{n}\right| \leqslant\left|\Delta x_{n}\right|+\varepsilon_{n}\left|g_{n}\left(X_{n}\right)\right| \text {. }
$$

Writing $\left|\varepsilon_{n}-\varepsilon\right|=O\left(\varepsilon^{2}\right)$ in the form $\varepsilon(1-M \varepsilon) \leqslant \varepsilon_{n} \leqslant \varepsilon(1+M \varepsilon)$ gives

$$
\varepsilon(1-M \varepsilon)\left|\psi_{n}\right| \leqslant\left|\Delta x_{n}\right|+\varepsilon(1+M \varepsilon)\left|g_{n}\left(X_{n}\right)\right| \text {. }
$$

With $\varepsilon^{*}$ small enough to satisfy $M \varepsilon^{*} \leqslant 1 / 2$,

$$
\varepsilon\left|\psi_{n}\right| \leqslant 2\left|\Delta x_{n}\right|+3 \varepsilon\left|g_{n}\left(X_{n}\right)\right|
$$

Our first estimate is:

$$
\varepsilon\left|\Psi_{n}\right| \leqslant 2 k\left|\Delta X_{n}\right|+3 \varepsilon k\left|G_{n}\left(X_{n}\right)\right|
$$

Summing up in (2),

$$
X_{n}=X_{m}+\varepsilon \sum_{j=m}^{n-1} G_{j}\left(X_{j}\right)+\varepsilon \sum_{j=m}^{n-1} \Psi_{j}+\sum_{j=m}^{n-1}\left(E_{j}-\varepsilon I\right)\left(G_{j}\left(X_{j}\right)+\Psi_{j}\right),
$$

so

$$
\varepsilon\left|\sum_{j=m}^{n-1} \Psi_{j}\right| \leqslant\left|X_{n}\right|+\left|X_{n}\right|+\varepsilon \sum_{j=m}^{n-1}\left|G_{j}\left(X_{j}\right)\right|+\sum_{j=m}^{n-1} M \varepsilon^{2}\left(\left|G_{j}\left(X_{j}\right)\right|+\left|\Psi_{j}\right|\right) .
$$

Stability in the sense of Definition 2 means that given any $\bar{\eta}>0$, there is a $\delta(\bar{\eta})>0$ such that for all sufficiently small $\varepsilon>0$, i.e., less than $\varepsilon^{*}(\bar{\eta}),\left|X_{n_{0}}\right|<\delta$ implies $\left|X_{j}\right|<\bar{\eta} / 2$, $\left|\Delta X_{j}\right|<\bar{\eta}$, and $\left|G_{j}\left(X_{j}\right)\right|<\bar{\eta}$ for $j \geqslant n_{0}$, the last following from the equicontinuity. Using this, $M \varepsilon^{*} \leqslant 1 / 2$, and (7) in (8) gives

$$
\varepsilon\left|\sum_{j=m}^{n-1} \Psi_{j}\right| \leqslant \bar{\eta}+\bar{\eta} \varepsilon\left(1+\frac{1}{2}(3 k+1)+2 M k\right)|n-m|, \quad n_{0} \leqslant m, n .
$$

To complete the proof, let arbitrary $\eta>0$ be given. Choose $\bar{\eta}$ and its accompanying $\delta$ and $\varepsilon^{*}$ so that $\left(1+\frac{1}{2}(3 k+1)+2 M k\right) \bar{\eta} \leqslant \eta$. We have

$$
\varepsilon\left|\sum_{j=m}^{n-1} \Psi_{j}\right| \leqslant \eta+\eta \varepsilon|n-m|
$$

which is equivalent to (6). 
Example 4. Any sequence $\left\{\xi_{j}\right\}_{j=0}^{\infty}$ whose partial sums $\sum_{j=m}^{n-1} \xi_{j}$ are uniformly bounded, like $\xi_{j}=a(\sin j)+b(\cos j)$ or $\xi_{j}=(-1)^{j}$ satisfies $(3)$ with $\|\cdot\|$ the Euclidean norm.

Let $\mathrm{E} \xi$ denote the expected (average) value of a random variable $\xi$ and $\|\xi\| \equiv \sqrt{\mathrm{E}|\xi|^{2}}$. All sequences of random variables, orthogonal and bounded in the $\left(L_{2}\right)$ sense that $\mathrm{E} \xi_{j}^{T} \xi_{k}=0, j \neq k$, and $\sup _{n} \mathrm{E}\left|\xi_{n}\right|^{2}<\infty$, satisfy (3) with this norm. An example is the coin-tossing sequence of Example 3. The orthogonality condition can be weakened, but the choice of norm is critical. The coin-tossing random variables do not satisfy (3) if we use absolute value instead of the $L_{2}$ norm and require that (3) holds along almost every sample path. This is due to the almost certain occurrence of arbitrarily long head (tail)-runs which destroy the uniform smallness of the averages [2, p. 42], ruling out (4). A deterministic sequence which imitates this long head-tail run property is $\sin \sqrt{n}$. The long-run average value of $\sin \sqrt{n}$ is zero, but its increasingly slow oscillations prevent the uniformity property implied by (4). A remark on this follows.

Remark. We have answered the question posed in the remark following Example 3. Evidently, the "random" fluctuations of $\sin n, n=0,1, \ldots$, satisfy a stronger law of averages, (4)-(6), than the one obeyed by a tossed coin or the increasingly lazy swings of $\sin \sqrt{n}$. This law of averages is necessary for stability. In the next section we show that it is also sufficient.

2. Necessary and sufficient condition for stability. Now we show that (6) is sufficient for stability in (2) in the sense of Definition 2. Some regularity conditions on $\left\{G_{n}(\cdot)\right\}_{n=0}^{\infty}$ are required, one of which, (A2) below, builds in stability in the absence of perturbation. These are:

(A1) Uniform Lipschitz equicontinuity of $\left\{G_{n}(\cdot)\right\}_{n=0}^{\infty}$ on $\mathbf{R}^{k}$, with Lipschitz constant $L>0,\left|G_{n}(X)-G_{n}(Y)\right| \leqslant L|X-Y|, X, Y \in \mathbf{R}^{k}, n=0,1, \ldots$

(A2) A condition using the classical Liapunov function $|X|^{2} / 2,\left\langle G_{n}(X), X\right\rangle \leqslant-\rho|X|^{2}$ $+a_{1}|X|+a_{2}, X \in \mathbf{R}^{k}, n=0,1, \ldots$, where $\rho>0$ and $a_{1}, a_{2} \geqslant 0$ are constants. (A1) and (A2) can be weakened considerably, but with addition of distracting and inessential detail in the proof of Theorem 2, below. We could use

(B1) Generalized Lipschitz continuity $\left|G_{n}(X)-G_{n}(Y)\right| \leqslant L\left(|X-Y|+|X-Y|^{\theta}\right)$, $0<\theta<1$.

(B2) $|X|^{2} / 2$ is replaced by a quadratic form, or a function that may be approximated by a quadratic form.

For simplicity we stay with (A1) and (A2).

Our method is to embed (2) in a differential equation which features additional perturbations due to the embedding. There are a number of reasons for taking this approach. For one thing, integration by parts, which we will use repeatedly, still seems more familiar and automatic than summation by parts. There is a similar contrast between the exponential integrating factor and its discrete analog. More importantly, as $\varepsilon \rightarrow 0$ in (2) (recall our running assumption that $\left|\varepsilon_{n}^{j}-\varepsilon\right|=O\left(\varepsilon^{2}\right)$ ) the $\Delta X_{n}=X_{n+1}-X_{n}$ are looking more and more like the infinitesimal adjustments of a differential equation. Our difference equations are literally evolving into a differential equation and we want to bring this evolution to the surface in the proof of Theorem 2. Our interpolation method gives maximum flexibility. Readers who are interested in differential equations will see that the 
analog of (6) in continuous time, (22), is sufficient for stability in the differential equation (11) below, without perturbations $p_{1}$ and $p_{2}$.

Let $[t]$ denote the greatest integer not greater than $t$. Define the piecewise constant interpolations

$$
\bar{X}(t) \equiv X_{[t]}, \quad \bar{\Psi}(t) \equiv \Psi_{[t]}, \quad \bar{G}(t, \cdot) \equiv G_{[t]}(\cdot), \quad t \geqslant 0,
$$

and piecewise linear interpolation

$$
X(t) \equiv(t-n) X_{n+1}+(n+1-t) X_{n}, \quad n \leqslant t<n+1 .
$$

System (2) becomes

$$
\dot{X}(t)=\varepsilon \bar{G}(t, X)+\varepsilon \bar{\Psi}+p_{1}+p_{2}, \quad t \neq n,
$$

where $p_{1}=\left(E_{[t]}-\varepsilon I\right)(\bar{G}(t, \bar{X})+\bar{\Psi})$ and $p_{2}=\varepsilon(\bar{G}(t, \bar{X})-\bar{G}(t, X))$. It is useful to consider the sources and orders of magnitude of errors $p_{1}$ and $p_{2}$. Error $p_{1}$ originates from our interest in seeing how much $\varepsilon_{n}^{j}$ can fluctuate, and the matrix $E_{[t]}-\varepsilon I$ has higher order $O\left(\varepsilon^{2}\right)$ terms along its diagonal. Error $p_{2}$ arises because we are approximating a piecewise constant path with a piecewise linear one. It looks ready for the Lipschitz condition and an estimate on $|\bar{X}(t)-X(t)|$ which verifies $p_{2}$ is also $O\left(\varepsilon^{2}\right)$.

Lemma 2. For sufficiently small $\varepsilon^{*}>0$ and all $0<\varepsilon<\varepsilon^{*}$, the piecewise constant and linear interpolations satisfy

$$
\begin{gathered}
|\bar{X}(t)| \leqslant 2|X(t)|+4 \varepsilon|\bar{\Psi}(t)|, \\
|\bar{X}(t)-X(t)| \leqslant 3 \varepsilon(L|X(t)|+|\bar{\Psi}(t)|), \quad t \geqslant 0 .
\end{gathered}
$$

Proof. Write $\varepsilon_{n}^{\prime} \leqslant \varepsilon(1+M \varepsilon)$ and let $\varepsilon^{*}=\min (1 /(2 M), 1 /(4 L))$ where $L$ is the Lipschitz constant of (A1).

$$
\begin{aligned}
|\bar{X}| & \leqslant|X|+|\bar{X}-X| \\
& \leqslant|X|+\varepsilon(1+M \varepsilon)(|\bar{G}(t, \bar{X})|+|\bar{\Psi}|) \\
& \leqslant|X|+2 \varepsilon(L|\bar{X}|+|\bar{\Psi}|) .
\end{aligned}
$$

Solving for $|\bar{X}|$ in (15), using the fact that $1-2 \varepsilon L \geqslant 1 / 2$, gives (12).

The second expression on the r.h.s. of (14) is a bound for $|\bar{X}-X|$. Working with that,

$$
|\bar{X}-X| \leqslant \frac{3}{2} \varepsilon(L|\bar{X}|+|\bar{\Psi}|) \leqslant \frac{3}{2} \varepsilon(2 L|X|+(4 \varepsilon L+1)|\bar{\Psi}|)
$$

gives (13).

It will turn out that errors $p_{1}$ and $p_{2}$ are trivial, to be dispensed with almost immediately in the proof of Theorem 2 below. The real object of interest is the differential equation $\dot{X}=\varepsilon \bar{G}(t, X)+\varepsilon \bar{\Psi}, t \neq n$.

Our basic technical device is an integral inequality in the spirit of Gronwall's Lemma (in fact, it is a true generalization, but we won't pursue that point). Although the proof of Lemma 3 is almost trivial, (16), below, serves as an effective accounting device in the proof of Theorem 2 . 
Lemma 3. Let $m_{\gamma}, j=1,2, \ldots, r$, denote finite measures on $\left[t_{0}, \infty\right)$, and let $f$ be a nonnegative function integrable on finite intervals with respect to every $m_{\gamma_{i}}$. Suppose that

$$
f(t) \leqslant \alpha+\beta \sum_{j=1}^{l} \int_{t_{0}}^{t} f^{q_{i}}(s) d m_{\gamma_{j}}+\bar{\varepsilon} \sum_{j=l+1}^{r} \int_{t_{0}}^{t} f(s) d m_{\gamma_{i}}, \quad t_{0} \leqslant t,
$$

where $\alpha, \beta \geqslant 0$ are constants and $0 \leqslant q_{j} \leqslant 1 / 2$. If the $m_{\gamma}\left[t_{0}, \infty\right)$ are uniformly finite for all multi-index values $\gamma_{j}$, and $\bar{\varepsilon}>0$ can be taken sufficiently small, then

$$
\sup _{t \geqslant t_{0}} f(t)=O(\alpha+\beta) \text {. }
$$

Proof. We use the well-known inequality $x y \leqslant c x^{p}+\frac{1}{c} y^{q}$ for $x, y \geqslant 0, c \geqslant 1, q \geqslant 2$, and $\frac{1}{p}+\frac{1}{4}=1$ in (16) with $x=1, y=f^{4}(s), q=1 / q_{j}$. As a result

$$
f(t) \leqslant \alpha+\beta c l B+\frac{\beta}{c} \sum_{j=1}^{l} \int_{t_{0}}^{t} f(s) d m_{\gamma_{j}}+\bar{\varepsilon} \sum_{j=l+1}^{r} \int_{\left.t_{0}\right)}^{t} f(s) d m_{\gamma_{j}}, \quad t_{0} \leqslant t,
$$

where $m_{\gamma_{1}}\left[t_{0}, \infty\right) \leqslant B<\infty$ for all $\gamma_{j}$. The integrability condition on $f$ and (16) imply that $f$ is bounded on finite intervals. Let arbitrary $T>t_{0}$ be given.

$$
f(t) \leqslant \alpha+\beta c l B+\left(\frac{\beta}{c}+\bar{\varepsilon}\right) r B \sup _{t_{0} \leqslant t \leqslant T} f(t) .
$$

Choose $\bar{\varepsilon}$ sufficiently small and $c$ sufficiently large $\left(c \geqslant 1\right.$ is arbitrary) so that $\left(\frac{\beta}{c}+\bar{\varepsilon}\right) r B$ $<1$. Taking the supremum on the l.h.s. of (18) over $t$ in $\left[t_{0}, T\right]$ and consolidating terms gives

$$
f(t) \leqslant \frac{\alpha+\beta c l B}{1-r B\left(\bar{\varepsilon}+\frac{\beta}{c}\right)}, \quad t_{0} \leqslant t \leqslant T .
$$

Since $T$ is arbitrary, (17) follows from (19).

Remark. In our actual use of Lemma 3 we will be able to take $\bar{\varepsilon}$ arbitrarily small. Since $c$ can be fixed arbitrarily large, it is assured that $O(\alpha+\beta)$ in (17) is independent of $\bar{\varepsilon}$.

Theorem 2. Let (A1) and (A2) hold. Suppose that for any $\eta>0$ there exist $\delta(\eta)>0$ and $\varepsilon^{*}(\eta)>0$ such that $\left|X_{n_{0}}\right|<\delta$ and $0<\varepsilon<\varepsilon^{*}$ imply (6). Then (i) if $a_{1}=a_{2}=0$ the zero solution of (2) is stable in the sense of Definition 2, otherwise (ii) solutions of (2) remain bounded as $n \rightarrow \infty$.

Proof. To avoid an overly technical appearance, we first outline the simple strategy. Differential equation (11) will be used to build an inequality of the form (16) with $V(t) \equiv|X(t)|^{2} / 2$ playing the role of $f$ there. Even though (11) is not defined on the integers, standard theorems in analysis (e.g., use of the right-handed derivative) allow us to treat (11) as a conventional ODE. Since the ability of $X_{n}$ to move away from the origin is measured by $V(t)$, we will monitor the size of all contributions to $\alpha$ and $\beta$ in (16)-(17) and verify that they can be held arbitrarily small. Thus, solutions $X_{n}$ which "start close, stay close" to the origin, and that is stability.

Starting with (11), using Schwarz's inequality on the terms involving $p_{1}, p_{2}$,

$$
\begin{gathered}
\dot{V}(t)=\langle X, \dot{X}\rangle \leqslant \varepsilon\langle X, \bar{G}(t, X)\rangle+\varepsilon\langle X, \bar{\Psi}\rangle+|X|\left|p_{1}\right|+|X|\left|p_{2}\right|, \quad t \neq n . \\
\dot{V}+2 \rho \varepsilon V \leqslant|X|\left|p_{1}\right|+|X|\left|p_{2}\right|+\varepsilon\langle X, \bar{\Psi}\rangle
\end{gathered}
$$


follows from (A2) with $a_{1}=a_{2}=0$ (we begin the proof of (i)). It was claimed earlier that $p_{1}, p_{2}$ would give the least amount of trouble and we show this now.

Using our assumptions that $\left|\varepsilon_{n}^{j}-\varepsilon\right|=O\left(\varepsilon^{2}\right), \varepsilon|\bar{\Psi}| \leqslant \eta(1+\varepsilon)((6)$, with $n=m+1)$. and Lemma 2 gives $|X|\left(\left|p_{1}\right|+\left|p_{2}\right|\right)=\varepsilon(O(\eta) \sqrt{V}+O(\varepsilon) V)$. Since $\varepsilon$ can become arbitrarily small, we set $\rho=1 / 2$ without loss of generality and obtain

$$
\dot{V}+\varepsilon V \leqslant \varepsilon(O(\eta) \sqrt{V}+O(\varepsilon) V+\langle X, \bar{\Psi}\rangle) .
$$

Integrate across (20) using the integrating factor $\exp (\varepsilon t), t \geqslant t_{0}=n_{0}$, to get

$$
\begin{array}{rl}
V(t) \leqslant V\left(t_{0}\right) \exp \left(-\varepsilon\left(t-t_{0}\right)\right)+O(\eta) \int_{t_{0}}^{t} \overline{V(s)} d m_{\gamma_{1}} & O(\varepsilon) \int_{t_{0}}^{t} V(s) d m_{\gamma_{1}} \\
& +\int_{t_{0}}^{t}\langle X, \bar{\Psi}\rangle d m_{\gamma_{1}},
\end{array}
$$

where $d m_{\gamma_{1}}$ denotes the Riemann-Stieltjes differential $\varepsilon \exp (-\varepsilon(t-s)) d s$. The two parameters of the multi-index $\gamma=(\varepsilon, t)$ are $\varepsilon>0$ and $t \geqslant t_{0}$. For every $\gamma$-value we have $m_{\gamma_{1}}[a, \infty)=\int_{[a, \infty) \cap\left[t_{0}, t\right]} d m_{\gamma_{1}} \leqslant 1$. Thus the $d m_{\gamma_{1}}$, abbreviated by $d m$ in the sequel, are a family of uniformly finite measures on $\left[t_{0}, \infty\right)$. Now suppose that the last integral on the r.h.s. of (21) were missing. In that case, we would be looking at an inequality precisely of the form (16) with $\alpha=V\left(t_{0}\right), \beta=O(\eta)$, and $\bar{\varepsilon}=O(\varepsilon)$. Let $\bar{\eta}>0$ of Definition 2 be given. Accordingly, choose $\eta(\bar{\eta}), \delta$, and $\varepsilon^{*}$ so that the r.h.s. of (17) is less than $\bar{\eta}^{2} / 2$. The implication is stability in the sense of Definition 2.

The rest of the proof consists of decomposing the last integral in (21), and here is where Lemma 3 pays dividends. More integrals will appear on the r.h.s. of (21) as we work with the last integral. Each time we encounter a new one, we verify that the numerical coefficient which multiplies it contributes an arbitrarily small amount to $\alpha$ or $\beta$ in (16), that is, $O(\eta)$. The stability will follow just as it did at the end of the last paragraph, from (17).

To begin the decomposition, consider a typical term in the inner product, $I_{0}=$ $\int_{t_{0}}^{t} x(s) \bar{\psi}(s) d m$. Integrate by parts, with $v(s)=-\int_{s}^{t} \bar{\psi}(\tau) d \tau, u(s)=x(s) \varepsilon \exp (-\varepsilon(t-s))$. Henceforth, let $C(s, t)$ denote $\int_{s}^{t} \bar{\psi}(\tau) d \tau$. We get $I_{0}=I_{1}+I_{2}+I_{3}$, where

$$
\begin{aligned}
& I_{1}=x\left(t_{0}\right) \exp \left(-\varepsilon\left(t-t_{0}\right)\right) \varepsilon C\left(t_{0}, t\right), \\
& I_{2}=\int_{t_{0}}^{t} x(s) \varepsilon C(s, t) d m, \\
& I_{3}=\int_{t_{0}}^{t} \dot{x}(s) C(s, t) d m .
\end{aligned}
$$

In working with $I_{1}, I_{2}, I_{3}$ we always use the following rule. When $\varepsilon|C(s, t)|$ appears, bound it above with the interpolated expression of (9)-(6):

$$
\varepsilon|C(s, t)| \equiv \varepsilon\left|\int_{s}^{t} \bar{\psi}(s) d s\right| \leqslant \eta(1+\varepsilon(t-s)), \quad s \leqslant t .
$$

Similarly, write

$$
\varepsilon|\bar{\psi}| \leqslant 2 \eta \quad((9), \text { with } n=m+1)
$$

since we may as well take $\varepsilon^{*} \leqslant 1$. 
Consider $I_{1}$. Using (22) and the maximum value of the function $y e^{-y}(1 / e)$,

$$
\left|I_{1}\right| \leqslant\left|x\left(t_{0}\right)\right|(1+1 / e) \eta .
$$

Since $x\left(t_{0}\right)=x_{n_{0}}$ is bounded, $I_{1}=O(\eta)$. Henceforth we use the notation $I_{1}=O(\eta) \rightarrow \alpha$ to signal that, in this case, $I_{1}$ is contributing to or being incorporated in $\alpha$ in (16). The contribution is of order $O(\eta)$.

Consider $I_{2}$.

$$
\left|I_{2}\right| \leqslant \int_{t_{0}}^{t}|x(s)| \eta(1+\varepsilon(t-s)) d m \leqslant \sqrt{2} \eta \int_{t_{0}}^{t} \sqrt{V(s)} d m_{\gamma_{1}}+\sqrt{2} \eta \int_{t_{0}}^{t} \sqrt{V(s)} d m_{\gamma_{2}},
$$

where a new measure $d m_{\gamma_{2}}=\varepsilon(t-s) d m_{\gamma_{1}}=\varepsilon^{2}(t-s) \exp (-\varepsilon(t-s)) d s$ appears on the r.h.s. of (24). A routine calculation shows that not just this measure, but all such measures defined recursively by $d m_{\gamma_{n+1}}=\varepsilon(t-s) d m_{\gamma_{n}}$ are uniformly finite in $\gamma=(\varepsilon, t)$, assigning no greater "length" than $(n-1)$ ! to any subinterval of $\left[t_{0}, \infty\right)$. We meet $d m_{\gamma_{3}}$ at the very end of the proof. $I_{2}=O(\eta) \rightarrow \beta$ in (16).

Consider $I_{3}=\int_{t_{0}}^{t}\left(\varepsilon_{[s]} \bar{g}(s, \bar{X})+\varepsilon_{[s]} \bar{\psi}(s)\right) C(s, t) d m$. Break this up into two integrals $I_{4}+I_{5}$ with

$$
I_{4}=\int_{t_{0}}^{t} \varepsilon_{[s]} \bar{g}(s, \bar{X}) C(s, t) d m
$$

In $I_{4}$ we can certainly use (22), with $2 \eta$, on $\varepsilon_{[s]} C(s, t)$ (recall that $\varepsilon_{[s]} \leqslant \varepsilon(1+M \varepsilon)$ ) and the Lipschitz condition on $\bar{g}$, followed by (12) to get

$$
\left|I_{4}\right| \leqslant \int_{t_{0}}^{t} L(4|X|+8 \varepsilon|\bar{\Psi}|) \eta(1+\varepsilon(t-s)) d m
$$

$8 \varepsilon L|\bar{\Psi}|$ is bounded by $(23)$; therefore

$$
\left|I_{4}\right| \leqslant O(\eta)\left(\int_{t_{0}}^{t}(1+\sqrt{V}) d m_{\gamma_{1}}+\int_{t_{0}}^{t}(1+\sqrt{V}) d m_{\gamma_{2}}\right) .
$$

In our notation, $I_{4}=O(\eta) \rightarrow \alpha, \beta$ in (16).

Consider $I_{5}=\int_{t_{0}}^{t} \varepsilon_{[s]} \bar{\psi}(s) C(s, t) d m$. We show that $I_{5}$ contributes an even smaller $O\left(\eta^{2}\right)$ to $\alpha$ in (16). $I_{5}$ requires the final decomposition into $I_{6}+I_{7}$ with

$$
I_{6}=\int_{t_{0}}^{t}\left(\varepsilon_{[s]}-\varepsilon\right) \bar{\psi}(s) C(s, t) d m
$$

Since $\left|\varepsilon_{\mid s]}-\varepsilon\right| \leqslant M \varepsilon^{2}$, we can write

$$
\left|I_{6}\right| \leqslant \int_{t_{0}}^{t} M \varepsilon|\bar{\psi}(s)| \varepsilon|C(s, t)| d m
$$

and use (22), (23) to bound $\left|I_{6}\right|$ above by $O\left(\eta^{2}\right)\left(\int_{t_{0}}^{t} d m_{\gamma_{1}}+\int_{t_{0}}^{t} d m_{\gamma_{2}}\right)$. By the finiteness of these measures, $I_{6}=O\left(\eta^{2}\right) \rightarrow \alpha$ in (16).

As last integral

$$
I_{7}=\int_{t_{0}}^{t} \varepsilon \bar{\psi}(s) C(s, t) d m .
$$


Integrate $I_{7}$ by parts with $v(s)=-\frac{1}{2} C^{2}(s, t), u(s)=\varepsilon^{2} \exp (-\varepsilon(t-s))$ to get

$$
I_{7}=\frac{1}{2} \exp \left(-\varepsilon\left(t-t_{0}\right)\right)\left(\varepsilon C\left(t_{0}, t\right)\right)^{2}+\frac{1}{2} \int_{t_{0}}^{t}(\varepsilon C(s, t))^{2} d m
$$

A final use of $(22)$ on $(\varepsilon C(s, t))^{2}$, along with the boundedness of the function $y^{2} e^{-y}$ and measures $d m_{\gamma_{3}}=\varepsilon(t-s) d m_{\gamma_{2}}$, gives $I_{7}=O\left(\eta^{2}\right) \rightarrow \alpha$ in (16).

The decomposition of $I_{0}=\int_{t_{0}}^{t} x(s) \bar{\psi}(s) d m$, a typical element in the inner product expansion of the last integral in (21), is complete. There are only a finite number of such elements, hence, the magnitudes of $\alpha$ and $\beta$ in completed inequality (21)-(16), with $V$ playing the role of $f$, are: $\alpha=V\left(t_{0}\right)+O(\eta), \beta=O(\eta)$. Let arbitrary $\bar{\eta}>0$ of Definition 2 be given. The $\sup _{t \geqslant t_{0}} V(t)=\sup _{n \geqslant n_{0}}\left|X_{n}\right|^{2} / 2$ is bounded by the r.h.s. of (17): $O\left(V\left(t_{0}\right)+\eta\right)$. With a last check of (19) and the Remark after Lemma 3, take $\eta$, $\delta$, and $\varepsilon^{*}$ small enough to make this r.h.s. less than $\bar{\eta}^{2} / 2$. This proves (i).

Fortunately, the proof of (ii) is immediate. Reintroducing nonzero $a_{1}, a_{2}$ simply adds $\varepsilon O(1)(\sqrt{V}+1)$ to the r.h.s. of (20). After having done all of the above, we would still be left with the expression $I_{8}=\int_{t_{0}}^{t} O(1)(\sqrt{V}+1) d m$ contributing $O(1)$ to both $\alpha$ and $\beta$ in (16). The bound on $V(t)$ obtained from (17) is finite, but it is no longer necessarily small. The proof of Theorem 2 is complete.

Finally, we turn to the analog of asymptotic stability, the near-convergence of Definition 1. This happens if the condition on the perturbations is independent of $X_{n_{0}}$.

THEOREM 3. Let the conditions of Theorem 2 hold without any requirement that $\delta$ exist, in other words, $\delta \equiv+\infty$. If $a_{1}=a_{2}=0$, then $\lim _{\varepsilon \rightarrow 0} \varlimsup_{n \rightarrow \infty}\left|X_{n}(\varepsilon)\right|=0$ for all initial values $X_{n_{0}}$.

Proof. All notation is from the proof of Theorem 2. There, we established inequality (16) for $V(t)$ in the form

$$
\begin{aligned}
& V(t) \leqslant V\left(t_{0}\right) \exp \left(-\varepsilon\left(t-t_{0}\right)\right)+O(\eta)\left(1+\int_{t_{0}}^{t} \sqrt{V} d m_{\gamma_{1}}+\int_{t_{0}}^{t} \sqrt{V} d m_{\gamma_{2}}\right) \\
& +O(\varepsilon) \int_{t_{0}}^{t} V d m_{\gamma_{1}}
\end{aligned}
$$

and, via Lemma 3, an upper bound for $V$. Now that (6) is independent of the initial value, fix $X_{n_{0}}$ arbitrarily and obtain, as in Theorem $2, \sup _{t \geqslant t_{0}} V(t) \leqslant O\left(V\left(t_{0}\right)+\eta\right)$. Substitute this upper bound for $V$ in the r.h.s. of (25), relaxing it further. Take the lim sup as $t \rightarrow \infty$ across both sides of (25) to get $\overline{\lim }_{t \rightarrow x} V(t) \leqslant O(\eta+\varepsilon)$. Since $\eta$ and $\varepsilon$ are arbitrarily small, this implies $\lim _{\varepsilon \rightarrow 0} \varlimsup_{n \rightarrow \infty}\left|X_{n}(\varepsilon)\right|=0$.

3. Appendix. System (2) applies whenever $k$ decision-makers are adjusting production levels $X_{n}$, with uncertainty about each other's reactions embodied in the perturbations. Degrees of "restraint" in these reactions are possible via the adjustment speeds. What is the interaction of perturbation and restraint on the stability of equilibrium? What sort of randomness can deflect best-intended actions further and further from equilibrium? We 
found that the randomness of coin-tossing is impossible to damp out, but that does not mean that the $X_{n}$ move far from equilibrium, only that it is impossible to hold $X_{n}$ arbitrarily close. Here we give an example to show that excursions arbitrarily far from the origin can occur under perturbations which obey the law of large numbers, regardless of small $\varepsilon>0$ (however, see the computer simulations which follow).

Consider the difference equation of Example 2

$$
y_{n+1}=(1-\varepsilon) y_{n}+\varepsilon \sin \sqrt{n} \text {. }
$$

Adapting interpolation notation (10) to (26) gives

$$
\dot{y}(t)=-\varepsilon y(t)+\varepsilon \sin \sqrt{t}+\varepsilon(y(t)-\bar{y}(t))+\varepsilon(\sin \sqrt{[t]}-\sin \sqrt{t}),
$$

and integrating,

$$
\begin{aligned}
y(t)=y(\tau) \exp (-\varepsilon(t-\tau))+\int_{\tau}^{t} \sin \sqrt{s} d m+\int_{\tau}^{t}(y(s)-\bar{y}(s)) d m & \\
& +\int_{\tau}^{t}(\sin \sqrt{[s]}-\sin \sqrt{s}) d m, \quad \tau \leqslant t,
\end{aligned}
$$

where $d m=\varepsilon \exp (-\varepsilon(t-s)) d s$. Integrate $\int_{\tau}^{t}(\sin \sqrt{s}) \varepsilon e^{\varepsilon s} d s$ by parts with $u=\sin \sqrt{s}, v=$ $e^{\varepsilon s}$ to get

$$
\begin{aligned}
y(t)=y(\tau) \exp ( & -\varepsilon(t-\tau))+\left(\sin \sqrt{t}-(\sin \sqrt{t}) \exp (-\varepsilon(t-\tau))-\int_{\tau}^{t} \frac{\cos \sqrt{s}}{2 \varepsilon \sqrt{s}} d m\right) \\
& +\int_{\tau}^{t}(y(s)-\bar{y}(s)) d m+\int_{\tau}^{t}(\sin \sqrt{[s]}-\sin \sqrt{s}) d m, \quad \tau \leqslant t .
\end{aligned}
$$

Iterating $\left|y_{n+1}\right| \leqslant(1-\varepsilon)\left|y_{n}\right|+\varepsilon$ gives the bound $\left|y_{n}\right| \leqslant\left|y_{0}\right|+1$. The mean value theorem implies $|\sin \sqrt{[s]}-\sin \sqrt{s}| \leqslant 1 /(2 \sqrt{[s]})$. We also have $|y(s)-\bar{y}(s)| \leqslant \varepsilon(|\bar{y}(s)|+$ $|\sin \sqrt{[s]}|)$. In (27), these and the finiteness of the measures $m$ yield

$$
|y(t)-\sin \sqrt{t}| \leqslant\left(\left|y_{0}\right|+2\right) \exp (-\varepsilon(t-\tau))+\frac{1}{2 \sqrt{\tau-1}}(1+1 / \varepsilon)+\varepsilon\left(\left|y_{0}\right|+2\right) \text {. }
$$

The r.h.s. of (28) can be made arbitrarily small for sufficiently large $t$ as follows. Let arbitrary $\eta>0$ be given. Fix $\varepsilon>0$ sufficiently small so that $\varepsilon\left(\left|y_{0}\right|+2\right)<\eta / 3$. Now choose $\tau>0$ sufficiently large so that $(1+1 / \varepsilon) / 2 \sqrt{\tau-1}<\eta / 3$. Finally, take $T>0$ sufficiently large so that $t \geqslant T$ implies $\left(\left|y_{0}\right|+2\right) \exp (-\varepsilon(t-\tau))<\eta / 3$.

The fact that $\lim _{\varepsilon \rightarrow 0} \overline{\lim }_{t \rightarrow \infty}|y(t)-\sin \sqrt{t}|=0$ means that, regardless of small $\varepsilon$ and initial position, the trajectories of $y_{n}$ are becoming like those of $\sin \sqrt{n}$, moving persistently away from equilibrium. These excursions can be made as wide as one likes by replacing $\sin \sqrt{n}$ by $M \sin \sqrt{n}$ in (26) and the above arguments, large $M>0$.

In practical simulations, one observes behavior different from that emphasized in the preceding example. The figures below show the results of computer experiments in which we examine the trajectories of (2) with constant $E_{n}=E=\operatorname{Diag}\left(\varepsilon^{1}, \varepsilon^{2}\right)$ for decreasing values of $\varepsilon^{i}$, and $G_{n}(x, y)=G(x, y)=(-.7 x+.4 y, .6 x-.5 y)$. The unperturbed system 
is asymptotically stable in this case. For random disturbances we used $\Psi_{n}=$ $\left(\mathrm{RND}_{n}^{1}, \mathrm{RND}_{n}^{2}\right)$ where RND denotes the values supplied by a random number generator on the IBM-PC. In each of the figures $-5 \leqslant \mathrm{RND}_{n}^{i} \leqslant 5, i=1,2$, and the initial data $\left(x_{0}, y_{0}\right)=(20,-30)$. COUNTER denotes the number of iterations carried out. We ended these after becoming satisfied that the displayed orbit had settled into an unchanging configuration. The figures also show the final few $(x, y)$-values before termination.
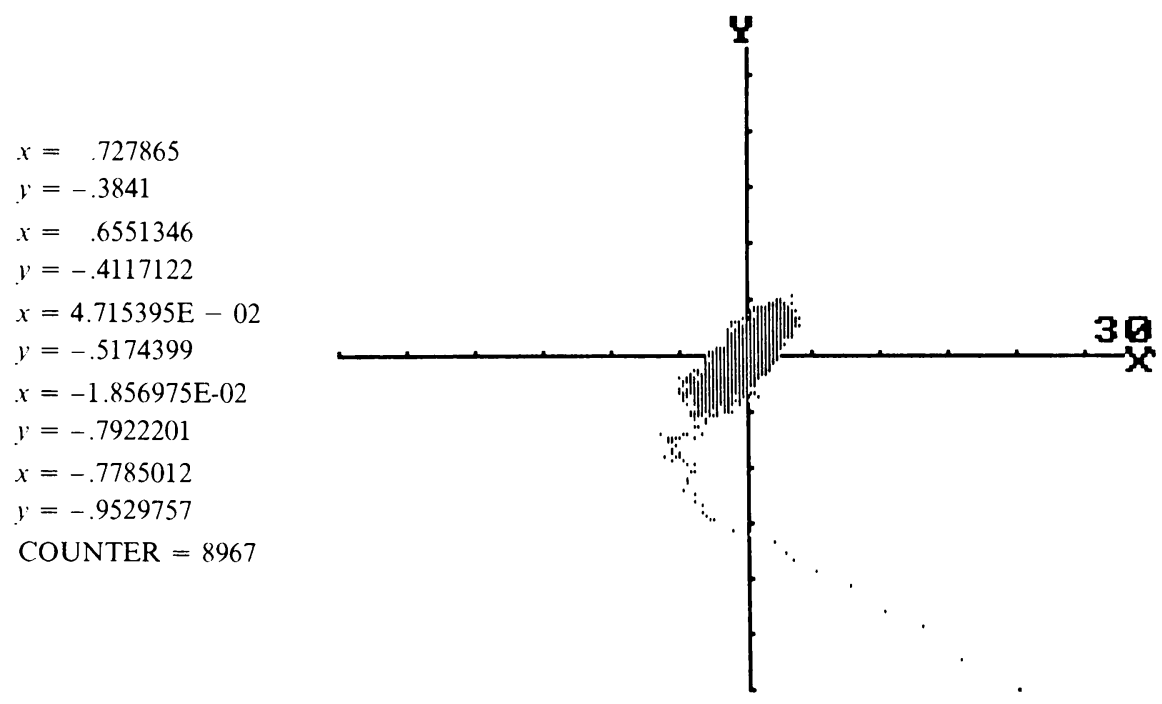

FIG. 1. $\varepsilon^{1}=.15, \varepsilon^{2}=.10$
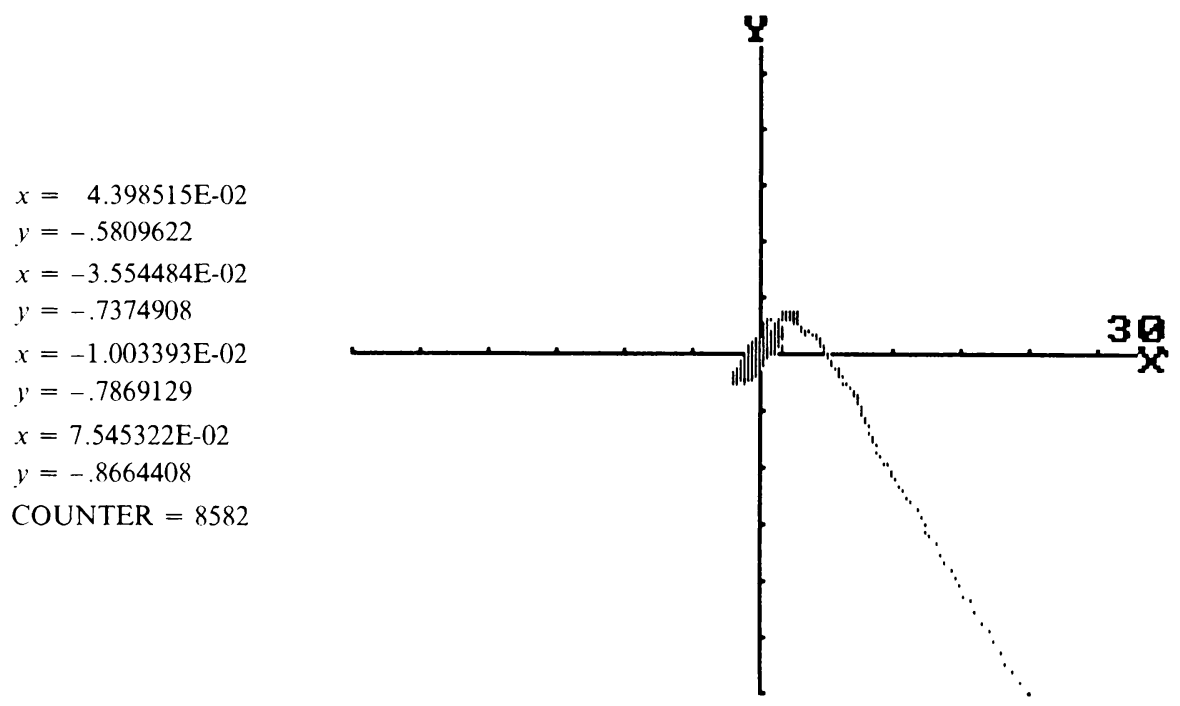

FIG. 2. $\varepsilon^{1}=.02, \varepsilon^{2}=.04$ 

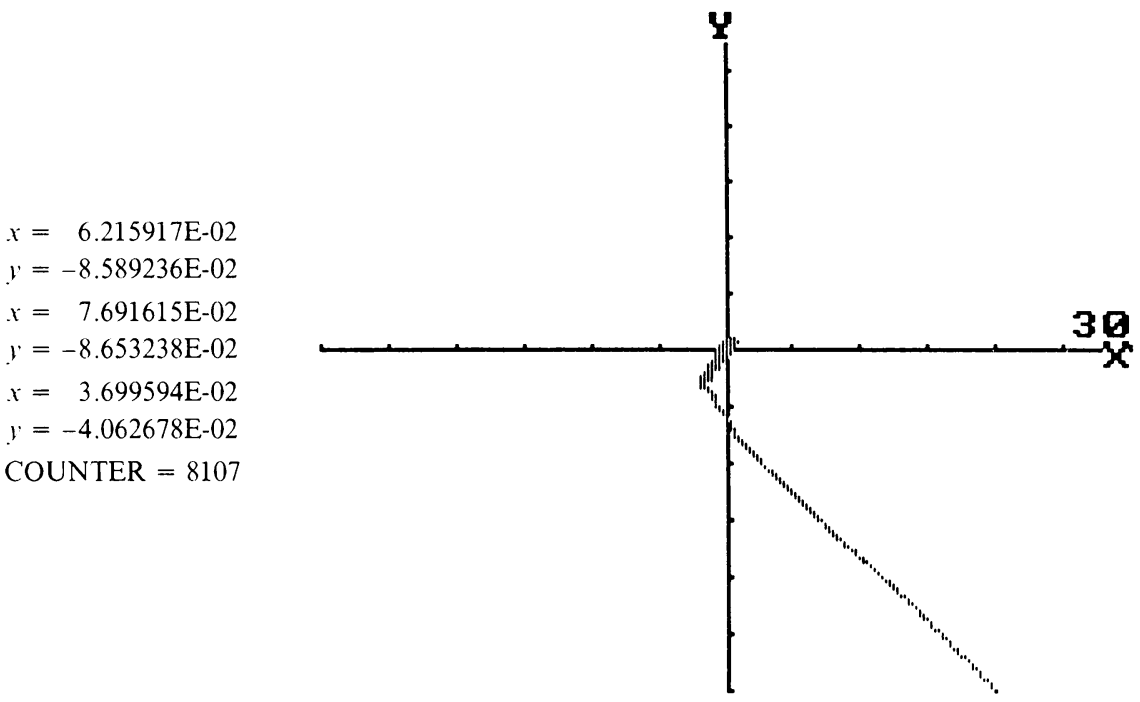

FIG. 3. $\varepsilon^{1}=.009, \varepsilon^{2}=.01$

The figures seem to show the near-convergence defined in (1). This is not surprising because, in the mean-square $\left(L_{2}\right)$ sense explained in Example 4, that is what is happening. The inequalities developed in the text remain valid in the $L_{2}$ norm, and the behavior of the PC's random number generator evidently approximates condition (4) in mean-square. The excursions away from the origin seem to become smaller and smaller as $\varepsilon \rightarrow 0$ and the "runs of bad luck" required to obtain truly large displacements from equilibrium are too long to be observed.

Acknowledgment. I wish to thank Professor John Fraleigh for his kind assistance in preparing the computer simulations, the members of the DE seminar at URI for their patience and suggestions, and an anonymous referee for constructive criticisms.

\section{REFERENCES}

[1] L. Brand, Differential and Difference Equations, Wiley, New York, 1966

[2] L. Breiman, Probability, Addison-Wesley, Reading, Mass., 1968

[3] W. Hahn, Üher die Anwendung der Methode von Ljapunov auf Differenzengleichungen, Math. Ann. 136, 430-441 (1958)

[4] J. M. Henderson and R. E. Quandt, Microeconomic Theory, McGraw-Hill, New York, 1971

[5] J. Hurt, Some stability theorems for ordinary difference equations, SIAM J. Num. Anal. 4, 582-596 (1967)

[6] J. P. LaSalle, Stability theory for difference equations, in Studies in Ordinary Differential Equations. J. K. Hale, ed. (MAA Studies in Mathematics, v. 14), 1-31 (1977)

[7] J. P. LaSalle, The Stability of Dynamical Systems, SIAM Regional Conference Series in Applied Mathematics, 1976 